\title{
Deshidratación de guayaba (Psidium guajava) en forma de rodaja mediante radiación solar en un secador directo
}

\author{
Dehydration of guava (Psidium guajava) in slice form by solar radiation in a direct \\ dryer
}

TENORIO-REYES, Susana†*, ROBLES-RAMÍREZ, Juan Daniel, CARRERA-ARELLANO, Ethson Uriel y GARCÍA-GONZÁLEZ, Juan Manuel

Universidad Autónoma de Zacatecas

ID $1^{\text {er }}$ Autor: Susana, Tenorio-Reyes / ORC ID: 0000-0002-2386-0092

ID $1^{\text {er }}$ Coautor: Juan Daniel, Robles-Ramírez / ORC ID: 0000-0001-6836-0924

ID $2^{\text {do }}$ Coautor: Ethson Uriel, Carrera-Arellano / ORC ID: 0000-0002-5323-6047, CVU CONACYT ID: 862754

ID $3^{\text {er }}$ Coautor: Juan Manuel, García-González, ORC ID: 0000-0001-7259-5021, CVU CONACYT ID: 346241

DOI: $10.35429 / J C P E .2019 .21 .6 .14 .18$

Recibido 09 Agosto, 2019; Aceptado 28 Noviembre, 2019

\section{Resumen}

El objetivo de este trabajo es disminuir el contenido de humedad de la guayaba (Psidium guajava) cortada en rodaja, a través de la radiación solar característica en la zona de la capital del estado de Zacatecas. Se empleó convección natural y convección forzada para alcanzar el objetivo de este estudio. La guayaba seleccionada proviene del municipio de Jalpa, Zacatecas. La humedad se evaluó con una balanza termogravimétrica OHAUS MB45, posteriormente se colocó las rodajas de la guayaba en charolas de malla polimérica con dimensiones de $34.2 \mathrm{~cm}$ de largo y $24 \mathrm{~cm}$ de ancho. Se introdujeron dentro de un secador transparente con dimensiones de $74 \mathrm{~cm} \times 80 \mathrm{~cm}$ de base y una altura frontal de $13 \mathrm{~cm}$ y posterior de $40 \mathrm{~cm}$. Dentro de los secadores se colocó un termómetro Checktemp 1 para evaluar la temperatura en el proceso de secado. La humedad inicial de la guayaba fue de $50.51 \%$, la humedad final obtenida en la convección natural fue de $8.08 \%$, mientras que en la convección forzada fue de $8.32 \%$, tras 8 horas de secado.

Deshidratado, Guayaba, Secado

\begin{abstract}
The objective of this work is to reduce the moisture content of the guava (Psidium guajava) cut in slices, through the solar radiation characteristic in the area of the capital of the state of Zacatecas. Natural convection and forced convection were used to achieve the objective of this study. The selected guava comes from the municipality of Jalpa, Zacatecas. Moisture was evaluated with an OHAUS MB45 thermogravimetric scale, then the guava slices were placed in polymer mesh trays with dimensions of $34.2 \mathrm{~cm}$ long and $24 \mathrm{~cm}$ wide. They were introduced inside a transparent dryer with dimensions of $74 \mathrm{~cm} \mathrm{x} 80 \mathrm{~cm}$ of base and a frontal height of $13 \mathrm{~cm}$ and posterior of $40 \mathrm{~cm}$. A Checktemp 1 thermometer was placed inside the dryers to evaluate the temperature in the drying process. The initial humidity of the guava was $50.51 \%$, the final moisture obtained in the natural convection was $8.08 \%$, while in the forced convection it was $8.32 \%$, after 8 hours of drying.
\end{abstract}

Dehydrated, Guava, Dried

Citación: TENORIO-REYES, Susana, ROBLES-RAMÍREZ, Juan Daniel, CARRERA-ARELLANO, Ethson Uriel y GARCÍA-GONZÁLEZ, Juan Manuel. Deshidratación de guayaba (Psidium guajava) en forma de rodaja mediante radiación solar en un secador directo. Revista de Energía Química y Física. 2019. 6-21: 14-18

\footnotetext{
* Correspondencia al Autor (Correo electrónico: jmgarcía@uaz.com.mx)

$\dagger$ Investigador contribuyendo como primer Autor
} 


\section{Introducción}

La guayaba es una fruta tropical de olor fragante, comúnmente de pulpa rojiza-rosada de sabor agridulce. Es conocida por sus amplias propiedades nutrimentales. Contiene una proporción elevada de agua y pocos hidratos de carbono, por lo que es una fruta recomendada en las personas que deben perder peso o mantenerlo Delgado Pérez, D. (2019). Además, de poseer una gran cantidad de vitamina C: (cinco veces más que la naranja) y es una buena fuente de antioxidantes y se le atribuyen propiedades como antiinflamatorio y anticancerígeno según lo reportado por Muñoz et al (2014) en la Tabla 1.

Lo reportado SAGARPA (2015) muestra que el mercado mundial de la Guayaba es de 6,000 Ton/año en promedio, siendo el primer importador el Reino Unido con cerca del 64\% del total de la producción mundial seguido de Estados Unidos y Canadá. Siendo México el cuarto abastecedor mundial de este producto.

A nivel nacional Zacatecas ocupa el tercer lugar de producción de guayaba con aproximadamente 37000 toneladas para el 2016, solo por debajo de Michoacán y Aguascalientes cuyas producciones en toneladas fueron de $117000 \mathrm{y}$ 53.5 respectivamente para el mismo año. Los productos procesados de guayaba importados a los Estados Unidos se clasifican en 3 categorías de acuerdo con el Snacks "Foods in U.S." (septiembre 2012):

- Pasta y puré, donde Brasil es el principal proveedor,

Preparados y conservas, donde México tiene una participación del 13\% después de Rep. Dominicana y Ecuador,

- Snacks, un procesado emergente de productos deshidratados donde México presenta un gran potencial debido a las características climáticas del territorio nacional.

El secado en alimentos es un proceso que involucra procesos de transferencia de energía y masa entre el entorno y el producto a tratar, donde factores como la geometría, propiedades químicas y propiedades físicas como la porosidad del producto, y condiciones ambientales como humedad relativa, temperatura y presión influyen en la calidad de deshidratado como lo describe Christie $J$ Geankoplis (2006).
Actualmente se conocen una gran variedad de métodos para retirare humedad a los alimentos que van desde secado a cielo abierto y secado en túneles los cuales involucran fenómenos de convección natural y forzada, hasta métodos químicos como la liofilización como lo menciona Colina Irezabal, M. (2010).

Comúnmente el secado de alimentosa gran escala se realiza al calentar un fluido encargado de transmitir esta energía a los alimentos y retirarles la humedad (normalmente aire) el cual tiene el inconveniente de utilizar combustibles fósiles para calentar el aire. Aunque actualmente están surgiendo nuevas tecnologías que concentran la energía solar para realizar los secados de manera eficiente sin el impacto ambiental que genera la quema de combustibles fósiles.

El objetivo de este trabajo es la deshidratación de guayaba, mediante el uso de secadores solares de caseta a través de convección natural $(\mathrm{CN})$ y convección forzada (CF). Así como evaluar su calidad mediante la preservación su color natural.

\begin{tabular}{|l|r|}
\hline $\begin{array}{l}\text { Valor energético y nutritivo } \\
\text { de } \mathbf{1 0 0} \text { g de guayaba cruda }\end{array}$ \\
\hline Kilocalorías & $57 \mathrm{~g}$ \\
\hline Proteínas & $0.82 \mathrm{~g}$ \\
\hline Grasas Totales & $0.6 \mathrm{~g}$ \\
\hline Colesterol & $0 \mathrm{~g}$ \\
\hline Fibra mineral & $5.4 \mathrm{~g}$ \\
\hline K (Potasio) & $290 \mathrm{mg}$ \\
\hline Hidratos de Carbono & $11.9 \mathrm{~g}$ \\
\hline
\end{tabular}

Tabla 1 Información nutrimental de $100 \mathrm{~g}$ de guayaba cruda.

\section{Materiales y métodos}

Se adquirió guayaba del sur del estado de Zacatecas en el municipio de Jalpa, Zac. La cual fue lavada y posteriormente cortada en rodajas de $4.075 \pm 0.5 \mathrm{~mm}$ con un espesor de $0.525 \mathrm{~mm}$, estas fueron colocadas en charolas de malla polimérica con dimensiones de $34.2 \mathrm{~cm}$ de largo y $24 \mathrm{~cm}$ ancho, registrando el peso de cada charola antes de exposición a la luz solar. Además, se determinó el porcentaje de humedad inicial de una de muestra de 0.5040 gr empleando una Termobalanza OHAUS. 
Las charolas se colocaron en dos secadores de acrílico transparente (Figura 1) con dimensiones de $74 \mathrm{~cm} \times 80 \mathrm{~cm}$ de base y una altura al frente de $13 \mathrm{~cm}$ y al fondo de $40 \mathrm{~cm}$. De los cuales, uno fue equipado con un ventilador de tres velocidades para provocar convección forzada en el proceso (Figura 1). Para medir la temperatura y la humedad se colocó un Termohigrómetro Profesional Instruments dentro de cada equipo.

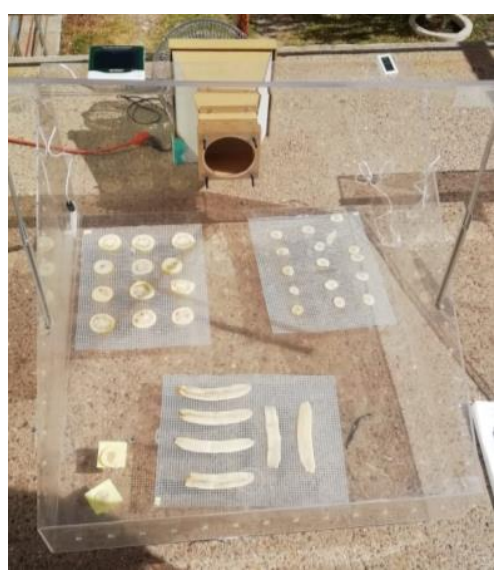

Figura 1 Cabina de acrílico con charolas

La metodología empleada para el secado y monitoreo se realizó de acuerdo con la propuesta de Méndez et al, (2017). De tal forma que, en el proceso de secado, las charolas fueron pesadas cada 15 minutos durante la primera hora y posteriormente cada 30 minutos. Además, para la determinación del color del producto se utilizó un colorímetro TPM desde el inicio hasta finalizar el secado en intervalos de una hora, mediante el sistema CIE-lab. Este sistema de evaluación del color trabaja en un plano tridimensional, con el eje "L" definiendo la luminosidad, el eje "a" va de verde a rojo y el eje "b" de azul hasta el amarillo, distribuidos como se muestra en la Figura 2.

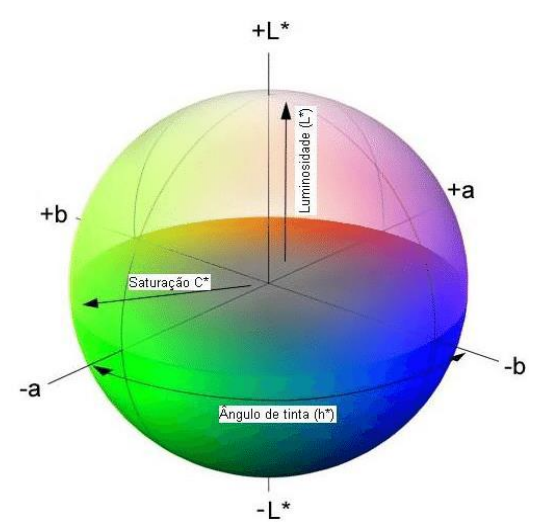

Figura 2 Diagrama del sistema CIElab

Fuente: https://www.researchgate.net/figure/Figura-13Sistema-de-coordenadas-de-cores-CIELab-adaptado-deCIELab-1976_fig4_310481467
La prueba de secado tuvo una duración de aproximadamente 8 horas, iniciando a las 10:00 h y concluyendo a las 16:00.

\section{Resultados}

Los resultados del colorimétrico indican que la guayaba deshidratada por convección forzada (Figura 3), tiene un color más claro que la guayaba secada en convección natural (Figura 4).
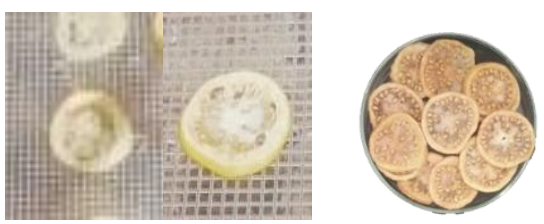

Figura 3 Desarrollo de las rodajas de la guayaba en secado por convección forzada
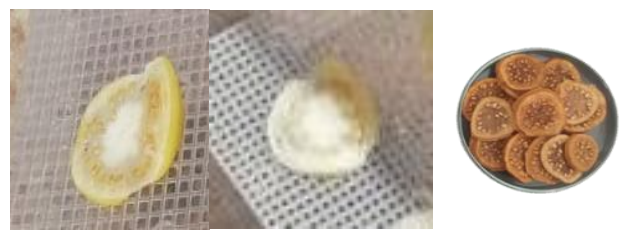

Figura 4 Desarrollo de las rodajas de la guayaba en secado por convección natural.

La tabla 2 muestra los resultados de la deshidratación de la guayaba en los secadores solares directos en convección natural y en convección forzada. Para poder comparar los resultados se empleó el mismo tiempo de deshidratación de $8 \mathrm{~h}$.

\begin{tabular}{|l|l|l|}
\hline Convección & $\mathrm{T}_{\text {prom }}\left({ }^{\circ} \mathrm{C}\right)$ & $\% \mathrm{H}_{\text {final }}$ \\
\hline Natural & $38.89 \pm 5.63$ & 8.08 \\
\hline Forzada & $34.11 \pm 3.24$ & 8.32 \\
\hline
\end{tabular}

Tabla 2 Resultados de la deshidratación de rodajas de guayaba en convección natural y forzada.

En la gráfica 1 se muestra muestran la pérdida de agua en peso en función del tiempo de deshidratación de la guayaba, tanto en convección natural, como en convección forzada

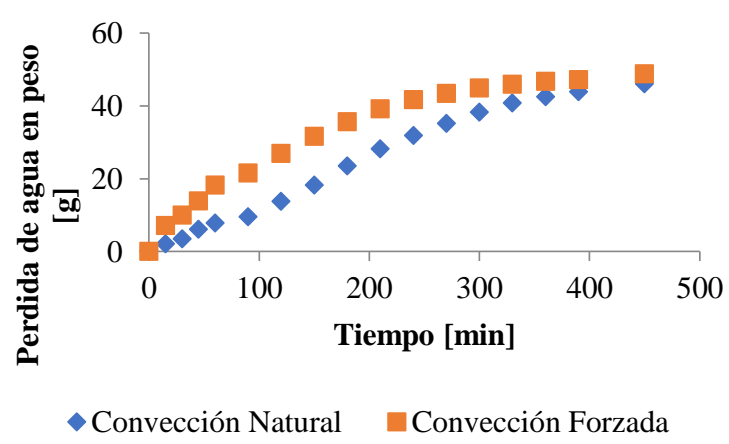

Gráfica 1 Pérdida de agua en peso durante el tiempo de secado

TENORIO-REYES, Susana, ROBLES-RAMÍREZ, Juan Daniel, CARRERA-ARELLANO, Ethson Uriel y GARCÍA-GONZÁLEZ, Juan Manuel. Deshidratación de guayaba (Psidium guajava) en forma de rodaja mediante radiación solar en un secador directo. Revista de Energía Química y Física. 2019. 
De acuerdo a lo que se observa en el gráfico 2, se pierde mayor cantidad de agua en el régimen por convección forzada.

Por otra parte, en gráfica 2 se observa la comparación de los dos regímenes de operación, esto facilita la observación de la curva mejor definida de la convección forzada, la cual se debe al flujo constante de aire, suministrado por medio del ventilador al secador solar directo.

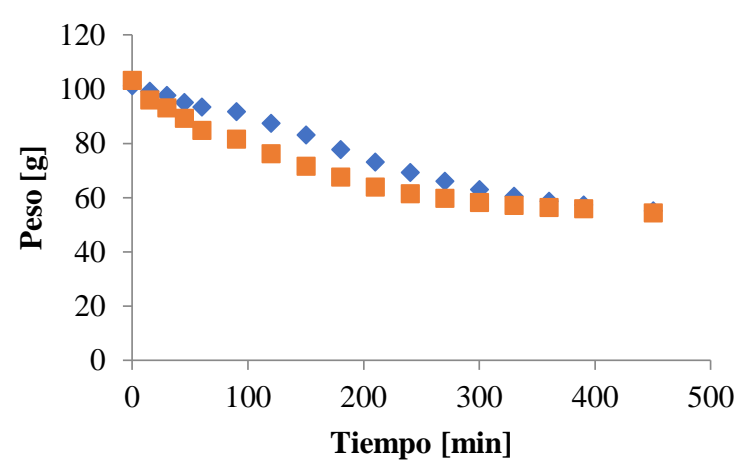

$\diamond$ Convección Natural Convección Forzada

Gráfica 2 Comparación de pérdida de peso contra tiempo en convección natural y forzada

Los datos obtenidos de la Estación Solarimétrica Zacatecas_04 en promedio durante la operación de deshidratado de la guayaba son: La irradiancia solar global fue de $560 \mathrm{~W} / \mathrm{m}^{2}$, la irradiancia solar directa fue de 540 $\mathrm{W} / \mathrm{m}^{2}$, la presión barométrica de $770.2 \mathrm{mbar}$, la velocidad del viento de $4.25 \mathrm{~m} / \mathrm{s}$ y dirección del viento $200^{\circ} \mathrm{SO}$, la temperatura de $25^{\circ} \mathrm{C}$ y la humedad relativa de $18.3 \%$. La velocidad del aire en el secador solar que operó en convección forzada fue de $3 \mathrm{~m} / \mathrm{s}$, proporcionando un caudal de $5.3 \times 10^{-2} \mathrm{~m}^{3} / \mathrm{s}$.

De acuerdo a lo observado se pierde 3.92 $\pm 1.36 \mathrm{~g}_{\mathrm{agua}} /{ }^{\circ} \mathrm{C}$ en el deshidratado de la guayaba utilizando secadores solares directos.

\section{Conclusiones}

De acuerdo al tiempo de secado no hay mucha diferencia en las operaciones de secado por convección natural y forzada. Aunque, en la convección forzada se tiene un secada más gradual, además de tener un mejor color del producto para su consumo. La mejor para el secado solar de guayaba en Zacatecas es con convección natural.

\section{Referencias}

Bueno T., F. (2017) "Desarrollo de un sistema de deshidratado de fruta de bajo costo mediante el control de temperatura para Pymes," Universidad Peruana de Ciencias Aplicadas (UPC).

Despacho Visual (22 de octubre de 2010). CIElab y CIElch. Obtenido de: https://despachovisual.wordpress.com/2010/10/ 22/cielab-y-cielch/

Estrada, H. et al (2018). Deshidratación Osmótica y Secado por Aire Caliente en Mango, Guayaba y Limón para la Obtención de Ingredientes Funcionales Obtenido de: https://scielo.conicyt.cl/scielo.php?script=sci_ar ttext\&pid=S0718-

07642018000300197\&lng=en\&nrm=iso\&tlng= en

FatSecret Platform API (04 de febrero de 2008). Guayaba, información nutricional. Obtenido de: https://mobile.fatsecret.com.mx/calor\%C3\%AD as-nutrici\% $\mathrm{C} 3 \% \mathrm{~B} 3 \mathrm{n} / \mathrm{gen} \% \mathrm{C} 3 \% \mathrm{~A} 9 \mathrm{rico} / \mathrm{guayaba}$

Gascón A., Muravnik N., Suliá P., Ventura L., Bressia C., D'Innocenzo S. (2006) "Tecnología de elaboración industrial de frutas y hortalizas deshidratadas" Universidad Nacional del Cuyo. 18-19

Geankoplis. (2006). "Procesos de Transporte y Operaciones Unitarias". 3era edición. Capítulo 9.

Méndez et al (2018) deshidratación de guayaba empleando secado de convección natural y forzada. por medio de la convección forzada el color resultante es más claro y su secado es más eficiente con una velocidad de aire de $0.55 \mathrm{~m} / \mathrm{s}$.

Rodriguez Villamil, D. (2016). Diseño de un colector solar de placa plana para la deshidratación de productos agroindustriales. (Licenciatura). Universidad Libre, Facultad de Ingeniería Mecánica. 
Pulido Blanco, V. C. (2019). Alternativas de manejo biológicas, culturales y químicas de los gusanos xilófagos anillador: carmenta theobromae (Busck, 1910)(Lepidoptera: Sesiidae); y taladrador: simplicivalva ampliophilobia Davis, Gentili-Poole \& Mitter, 2008 (Lepidoptera: Cossidae) de la guayaba (Psidium guajava L) en La Hoya del Río Suárez, Colombia.

Tenorio Reyes S., Robles Ramírez J.D., Carrera Arellano E.U., García González J.M., Carranza Concha J.."Deshidratación de pera en forma de rodaja en un secador solar directo". Journal of Energy, Engineering Optimization and Sustainability, Vol. 3 No. 1, 2019 